\title{
LONG-TERM HAEMODIALYSIS IN SPINAL CORD INJURED PATIENTS
}

\author{
By N. D. VAziri, M.D., F.A.C.P. \\ Division of Nephrology, University of California, Irvine, California, U.S.A.
}

Summary. The experience with chronic haemodialysis in 43 spinal cord injured patients is described. Spinal cord injured patients exhibited significant clinical and biochemical differences when compared with the ambulatory dialysis patients. Recognition of these characteristics can improve the quality of care rendered to these patients.

Key words: Spinal cord injury; Haemodialysis-long term.

\section{Introduction}

Both acute and chronic renal failure can occur in the course of spinal cord injury (SCI). Haemorrhagic shock or rhabdomyolysis associated with the accident causing SCI can lead to acute renal failure. Similarly acute renal failure can occur in SCI patients with the use of radiographic contrast media, various drugs such as aminoglycoside antibiotics, other nephrotoxic agents or those causing acute interstitial nephritis.

End-stage renal failure can also complicate the course of long-standing SCI due to chronic urinary tract infection, urolithiasis and renal amyloidosis. Before the advent and widespread availability of modern dialytic facilities the mortality associated with acute renal failure was extremely high and death was an inevitable consequence of end-stage renal dialysis (ESRD). With the use of dialytic treatments the mortality and morbidity associated with acute renal failure has been reduced considerably and death from chronic renal failure can be prevented. Until recently published data on the use of dialytic modalities in SCI patients were quite limited. The use of short-term haemodialysis and peritoneal dialysis was previously reported by Mitchell (I964) in a few SCI patients with acute renal failure. We recently published our data on 43 SCI patients with end-stage renal failure. treated with long-term haemodialysis for periods ranging from I to 60 months (Vaziri et al., I 982). Comparison of various clinical and biochemical parameters from our SCI-ESRD patients with those of the ambulatoryESRD patients revealed substantial differences. A summary of this and other works is provided.

\section{Patients and Methods}

\section{Patients}

Forty-three men, aged 26 to 75 years, with long-standing spinal cord injury and chronic renal insufficiency were included in the study. The aetiology

Address all correspondence to: N. D. Vaziri, M.D., Room C35I, Med. Sci. I., Division of Nephrology, Department of Medicine, University of California, Irvine, Calif. 927 1 7 , U.S.A. 
of renal failure was thought to be chronic pyelonephritis often accompanied by renal amyloidosis. A control group of ambulatory dialysis patients were also included for comparison. They were all men aged 33 to 83 years and had received dialysis prescriptions similar to those received by the SCI group.

\section{Technical Aspects}

Access to the blood stream was provided with surgical creation of Cimino arteriovenous fistulae or placement of polytetrafluoroethylene (PTFE) grafts in the majority of cases. Bovine carotid artery grafts and external arteriovenous shunts were used in some patients. The access was usually placed in the forearm and occasionally in the lower extremities. The internal blood access was usually created when the creatinine clearance fell below IO $\mathrm{ml} / \mathrm{min}$ or when BUN and/or creatinine concentrations reached 80 and $8 \mathrm{mg} / \mathrm{dl}$ respectively. This usually provided sufficient time for the access to mature (particularly for Cimino A-V fistulae) before initiation of haemodialysis treatments. When immediate dialysis was indicated the less desirable external shunts were placed or intermittent peritoneal dialysis was begun while awaiting the maturation of the internal access.

Indications for prompt initiation of dialysis therapy included: gastrointestinal symptoms, central nervous system manifestations, pericarditis, peripheral neuropathy, severe anaemia, platelet dysfunction with bleeding diathesis, marked azotaemia (BUN > IOO mg/dl), fluid overload unresponsive to $\mathrm{Na}$ restriction and diuretics, metabolic acidosis, hyperkalaemia and hyperphosphataemia.

Hollow fibre or parallel flow dialyzers with $\mathrm{I} \cdot 2-2 \cdot 5 \mathrm{~m}^{2}$ surface areas were used with single-pass dialysate delivery system and a glucose-free acetate-based dialysate. Most of the patients were dialyzed for 4 hours three times weekly although some patients received 6-hour-long or twice weekly treatments. Dialysate and blood flow rates were usually 500 and $200 \mathrm{ml} / \mathrm{min}$ respectively, although considerable variations in blood flow rate existed depending upon the haemodynamic and other considerations.

Anticoagulation was accomplished using systemic heparinization during dialysis in most instances. Regional heparinization was used in patients with pericarditis to avoid tamponade, those with active gastointestinal or other bleeding disorders and the post-operative cases. Fluid removal by ultrafiltration was accomplished by increasing the outflow resistance and was measured using bed-scales. The need for and the extent of ultrafiltration was determined by the status of fluid balance using interdialytic weight gain, peridialysis blood pressure, presence of oedema and cardiopulmonary status.

\section{Dietary Considerations and Medications}

No caloric or protein restrictions were prescribed. In fact many patients exhibiting nephrotic range proteinuria (due to renal amyloidosis) were encouraged to consume high protein diets. Fluid restriction was prescribed to limit the interdialytic weight gain to $I \cdot 2 \mathrm{~kg}$. When indicated (hypertension, oedema, cogestive heart failure) sodium intake was limited to about $2 \mathrm{~g}$ per day. Potassium restriction to $2-3 \mathrm{~g} /$ day was often necessary to 
maintain its predialysis serum level below $5.6 \mathrm{mg} / \mathrm{L}$. It should be noted that patients did not always fully comply with the dietary prescriptions.

Aluminium carbonate was given in 3 to 4 divided doses to control hyperphosphataemia. The dose was adjusted according to the predialysis serum phosphorus levels. Multivitamin preparations and folic acid (I $\mathrm{mg} /$ day) were prescribed for all patients. Iron preparations were given orally or parenterally (iron dextran) when iron deficiency was suspected. Blood transfusions were prescribed for symptomatic anaemia (dyspnoea, weakness, angina pectoris etc.). Oral or parenteral androgen preparations were also used to stimulate ery thropoiesis in many cases. Interdialytic hypertension was usually managed by sodium restriction and ultrafiltration. However, various antihypertensive agents had to be also used in some patients.

Bowel care consisting of glycerol suppositories and digital evacuation was given on off-dialysis days as needed.

\section{Results and Discussion}

\section{Clinical Features}

The interdialytic weight gain in the SCI patients $(0.7 \pm 0.8 \mathrm{~kg})$ was significantly $(\mathrm{p}<0.00 \mathrm{I})$ less than that observed in the ambulatory patients $(1 \cdot 7 \pm 1 \cdot 8 \mathrm{~kg})$. This was due to the higher urine output in the SCI group attenuating the degree of fluid retention between dialysis treatments. Consequently SCI patients required less severe fluid restriction than the ambulatory patients. The higher urine output in the SCI group despite comparable residual creatinine clearances reflects predominant tubulointerstitial involvement in these patients. Predialysis systolic blood pressure in the SCI group (I $54 \pm 22 \mathrm{mmHg}$ ) was significantly higher $(\mathrm{p}<0.05$ ) than that of the ambulatory group (I48 $\pm 25 \mathrm{mmHg}$ ). The respective values for diastolic pressure were $80 \pm 13$ and $75 \pm 12 \mathrm{mmHg}(\mathrm{p}=\mathrm{NS})$. The tendency for higher predialysis blood pressure despite lesser fluid retention in the SCI group as compared with the ambulatory group is of considerable interest. The observed disparity suggests the important role of peripheral vascular resistance in the genesis of hypertension in the SCI-ESRD patients.

\section{Biochemical Characteristics}

Predialysis serum concentrations of $\mathrm{Na}, \mathrm{K}$ bicarbonate and anion gap were significantly lower and chloride concentration was significantly higher in the SCI group than those found in the ambulatory patients. Similarly, predialysis serum creatinine concentration in the SCI group (8.2 \pm 3.4 $\mathrm{mg} / \mathrm{dl}$ ) was significantly lower $(\mathrm{p}<0.00 \mathrm{I})$ than that of the ambulatory patients $(15 \cdot 6 \pm 5 \cdot 1 \mathrm{mg} / \mathrm{dl})$. This was thought to reflect lower muscle mass and, thereby, endogenous creatinine production in the SCI patients. BUN and uric acid concentrations, however, were comparable in the two groups. The SCI patients had significantly lower serum albumin concentrations $(3.1 \pm 0.79 \mathrm{~g} / \mathrm{dl})$ than the ambulatory patients $(4.0 \pm 0.7 \mathrm{~g} / \mathrm{dl}, \mathrm{p}<0.00 \mathrm{I})$. This was felt to be due to the high incidence of nephrotic range proteinuria associated with renal amyloidosis in the SCI patients. In addition 
hepatic amyloidosis and nutritional factors could also have contributed to hypoalbuminaemia in these patients (Meshkinpour et al., 1982). Serum calcium concentrations in the SCI patients $(8 \cdot 6 \pm \mathrm{I} \cdot \mathrm{I} \mathrm{mg} / \mathrm{dl})$ was significantly lower $(p<0.00 \mathrm{I})$ than that of the ambulatory patients $(9.4 \pm 0.8$ $\mathrm{mg} / \mathrm{dl}$ ). This was thought to be in part due to lower protein bound calcium associated with hypoalbuminaemia in the SCI group. Serum-inorganic phosphorus level was also lower in SCI group. With dialysis serum concentrations of creatinine, urea nitrogen, uric acid, potassium and phosphorus fell while serum bicarbonate and calcium levels rose consistently. The per cent changes induced by dialysis were comparable in the two groups. Haemodialysis was, therefore, as effective in azotaemia control and maintaining acid-base and fluid balance in SCI patients as it was in the ambulatory individuals.

\section{Complications}

Symptomatic and asymptomatic hypotension occurred with considerable frequency during dialysis. This responded to the temporary reduction in blood flow rate and/or fluid administration. Mild to moderate and occasionally marked rise in blood pressure was noted despite ultrafiltration on some occasions. Intradialytic episodes of hypotension and hypertension were considerably more frequent in the SCI group as compared with the ambulatory dialysis patients. Headaches, nausea, and vomiting were commonly noted during dialysis and early post-dialysis periods. Painful muscle cramps associated with rapid fluid shifts or hypovolaemia, frequently encountered in the ambulatory patients during dialysis, were uncommon in the SCI patients. However, a diffuse painful muscle spasm involving lower extremities, abdominal muscles or, less commonly, the upper extremities were noted in 20 per cent to 30 per cent of the patients. This phenomenon is due to uninhibited spinal reflexes and could occur spontaneously or be provoked by a variety of conditions such as faecal impaction, rapid ultrafiltration during dialysis or other stimuli. Diazepam or baclofen [4-amino-3-(P-chlorophenylbutyric acid)], were used in an attempt to control severe muscle spasms with variable response. In addition, when identified the provocative factor(s) was eliminated. Worsening or occurrence of pruritus during dialysis treatments were recorded in several SCI patients.

\section{Psychosocial Aspects}

In depth data concerning the psychological profile of the SCI patients were not available. However, some degree of depression was felt to be commonly present. None of the SCI patients were employed and the majority were chronically hospitalized. Only one patient was on a home-dialysis programme. The degree of participation in self care during in-centre dialysis was negligible. The ambulatory patients, on the other hand, did considerably better with respect to all the above items. It is thus apparent that the combination of ESRD and SCI considerably impacts the pyschological aspects of the affected patients in a negative way.

In conclusion, dialysis is effective in sustaining life and controlling azotaemia, acid-base disorders and fluid and electrolyte abnormalities in 
patients with combined SCI and ESRD. The SCI patients, however, experience more intradialytic complications than ambulatory patients. In addition, their psychological adjustment is less favourable than that attained in ambulatory population. However, in the opinion of the author, these negative features should not preclude admission of such patients to maintenance dialysis programmes. This viewpoint is based on two considerations. As more experience is gained the prospect for better management of these patients will no doubt improve. Moreover, the decision concerning dialysis in ESRD is one of life or death, a decision which I believe clearly lies with the patient.

\section{RÉSUMÉ}

Les résultats de l'hémodialyse chronique dans 43 patients avec des blessures de l'épine dorsale sont décrits. Chez ces patients, comparés à des patients ambulatories, nous avons mis en évidence des différences cliniques et biochimiques. En tenant compte de ces charactéristiques, les soins de ces patients peuvent être améliores.

\section{ZUSAMMENFASSUNG}

Die Erfahrung mit chronischer Haemodialyse bei 43 Patienten mit Rückenmarkverletzungen ist beschrieben. Im Vergleich mit ambulanten Dialyse Patienten weisen Patienten mit Rückenmarkverletzungen bedeutende klinische und biochemische Unterschiede auf. An Hand dieser Erkenntnisse kann die Pflege dieser Patienten verbessert werden.

\section{REFERENCES}

MEShKinPoUR, H., VAZIRI, N. D. \& GoRDON, S. (I982). Gastrointestinal pathology in endstage renal disease associated with spinal cord injury. Amer. F. Gastroenterology, 77, $562-565$.

MitCHELL, M. D. (1964). The use of haemodialysis in renal failure complicating paraplegia. A report of five cases. Paraplegia, 2, 254-26I.

Vaziri, N. D., Bruno, A., Byrne, C., Mirahmadi, M. K., Nikakhtar, B., Gordon, S. and ZEINEN, L. (I982). Maintenance hemodialysis in end-stage renal disease associated with spinal cord injury. Artificial Organs, 6(I), I3-I6. 